\title{
Frühinfekt in der Endoprothetik
}

Mustafa Citak, Akos Zahar, Daniel Kendoff

\section{Einleitung}

Die Knie- und Hüftendoprothetik sind erfolgreiche Operationsverfahren mit exzellenten langfristigen Ergebnissen [17]. In Deutschland werden jährlich knapp 400000 endoprothetische Versorgungen pro Jahr durchgeführt. Aufgrund des demografischen Wandels wird die Zahl der primären und Revisionseingriffe in naher Zukunft weiter ansteigen. Konsequenterweise wird sich dies auch auf die Anzahl von postoperativen Komplikationen auswirken. Insbesondere die periprothetische Infektion (PPI) nach einem künstlichen Gelenk ist eine gefürchtete und schwer zu behandelnde Komplikation. Die Inzidenz nach primären Hüfttotalendoprothesen (HTEP) wird auf 1\% und nach primären Knietotalendoprothesen (KTEP) zwischen 1 und 2\% geschätzt $[7,18]$. Die Inzidenz nach Revisionsoperationen wird deutlich höher beschrieben [5, 23]. Somit liegt die Inzidenz nach einer Revisions-HTEP bei $14,8 \%$ und nach Revisions-KTEP bei $25,2 \%$ [5,23]. Diese Fakten zeigen, dass Revisionseingriffe aufgrund periprothetischer Infektionen trotz präoperativer präventiver Maßnahmen ein zunehmendes Problem sein werden. Weitere Herausforderungen sind die frühzeitige Diagnosestellung und die adäquate Therapiewahl, wobei sich die Therapiewahl zwischen einem Früh- und Spätinfekt unterscheidet.

\section{Einteilung der periprothetischen Infektion}

Es gibt diverse Möglichkeiten, die periprothetische Infektion zu klassifizieren; z. B. nach dem Zeitraum einer möglichen Kontamination und dem Auftreten erster Symptome oder nach der Ätiologie der Infektion, also nach den auslösenden Erregern. Im klinischen Alltag ist die Einteilung nach dem Zeitraum zwischen vermuteter Kontamination und dem Auftreten erster Symptome die am häufigsten verwendete Art [4, 27, 29].

Die Klassifikation von Tsukayama unterteilt die periprothetischen Infektionen in 4 Typen [27]. Typ 1 beschreibt den intraoperativen Keimnachweis der gewonnenen Proben im Rahmen einer aseptischen Wechseloperation. Die Unterscheidung zwischen Typ 2 und 3 richtet sich nach dem Zeitpunkt der Infektentstehung. Ein Zeitintervall von kleiner als 3 Wochen ist demnach ein Frühinfekt. Als (chronischer) Spätinfekt wird eine Infektion, die nach 3 Wochen auftritt, beschrieben. Eine Infektion durch hämatogene Keimaussaat infolge einer Barrierestörung beschreibt die Typ-4-Infektion [27]. Die Klassifikation nach Zimmerli und Trampuz beschreibt als Frühinfektion eine Infektion, die innerhalb von 3 Monaten nach einem Eingriff auftritt [29]. Als Spätinfekt wird eine Infektion beschrieben, die nach 24 Monaten nach einem künstlichen Gelenkeingriff vorkommt. Eine Infektion zwischen 3 und 24 Monaten wird als verzögerte Infektion eingestuft [29].

McPherson et al. entwickelten hingegen ein Staging-System für periprothetische Hüftgelenkinfektionen unter Berücksichtigung diverser Gesichtspunkte [20]. Hier sind insbesondere Kriterien wie Begleiterkrankungen des Patienten und Pathogenität des Erregers von Bedeutung. Die Klassifizierung kann den Chirurgen helfen, die Schwere der einzelnen Infektion zu beurteilen und eine patientenbezogene geeignete Behandlung zu wählen. Die Klassifikation wurde in der klinischen Praxis vor allem in den Vereinigten Staaten und in England verwendet [14, 21].

Nach den neuen Guidelines der „Musculoskeletal Infection Society“ (MSIS) wird eine Infektion als Frühinfektion klassifiziert, wenn sie innerhalb der ersten 4 Wochen nach der Operation auftritt. Alle Infektionen ab der 4. postoperativen Woche werden als chronische bzw. Spätinfektionen unterteilt [24].

\section{Klinik der periprothetischen Infektion}

Die klinischen Symptome sind bei akuten und chronischen Infekten sehr unterschiedlich. Die akute Infektion präsentiert sich oft mit einer fulminanten Verschlechterung der Funktion des betroffenen künstlichen Gelenkes, meistens begleitet von einem erheblichen Lokalbefund ( $\bullet$ Abb. 1). Der Gesundheitszustand des Patienten kann sich ebenfalls akut verschlimmern In einigen Fällen kann sich auch ein septisches Krankheitsbild zeigen.

Bei der chronischen Form hingegen haben die Patienten nur eher unspezifische Beschwerden. Bei folgenden Bedingungen muss man als behandelnder Arzt immer an eine periprothetische Infektion denken bzw. ausschließen: 


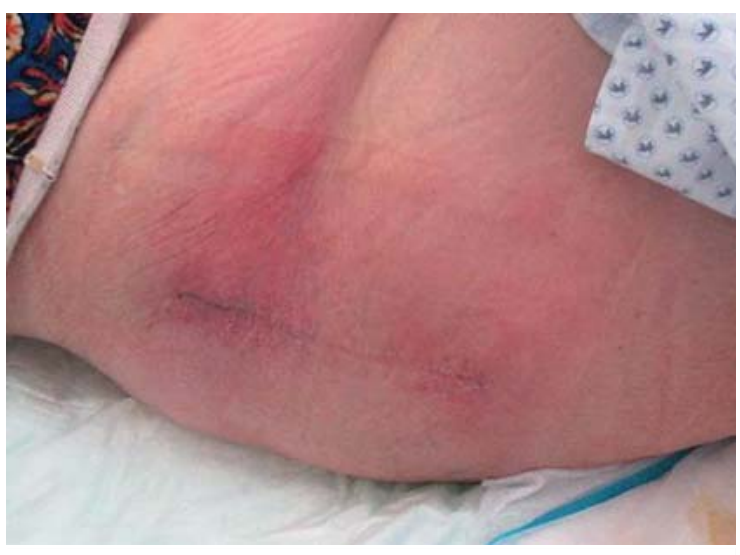

- Abb. 1 Akute Infektion einer Hüftprothese mit erheblicher lokaler Entzündungsreaktion, 3 Wochen nach primärer Implantation.

- Lockerung des künstlichen Gelenkes innerhalb des 1. Jahres nach Implantation

- Schmerzen sowie erhöhte Serumwerte des C-reaktiven Proteins (CRP) unklarer Ursache

- vorliegende B-Symptomatik (Nachtschweiß, Gewichtsverlust, Fieber)

Die ausführliche Anamnese im Rahmen der Nachsorgeuntersuchung nach einer elektiven Implantation eines künstlichen Gelenkes können wichtige Hinweise für die Diagnosestellung einer periprothetischen Infektion geben. Jeder Patient sollte hinsichtlich postoperativer Wundheilungsstörungen oder Hämatome gezielt gefragt werden.

\section{Definition der periprothetischen Infektion}

Im Jahr 2013 wurde im Rahmen des „International Consensus Meetings“ zur periprothetischen Gelenkinfektion in Philadelphia eine neue Definition der periprothetischen Gelenkinfektion erarbeitet [24]. Die Definition wurde von Vorstandsmitgliedern des „Musculoskeletal Infection Society“ (MSIS) und einer internationalen Expertengruppe von über 300 Delegierten erstellt. Die neue Definition ermöglicht sowohl den Klinikern als auch den Wissenschaftlern eine einheitliche und konsistente Diagnosestellung. Periprothetische Gelenkinfektionen werden demnach definiert, wenn ein Major-Kriterium und/oder 3 der 5 Minor-Kriterien vorliegen [24]:

Major-Kriterien:

- 2 positive periprothetische Kulturen mit phänotypisch identischen Organismen

- mit Gelenk kommunizierender Fistelgang
- Tab. 1 Unterschiedliche Referenzwerte für akute und chronische periprothetische Infektionen.

\begin{tabular}{|l|l|l|}
\hline $\begin{array}{l}\text { akute } \\
\text { Infektion }\end{array}$ & $\begin{array}{l}\text { chronische } \\
\text { Infektion }\end{array}$ \\
\hline CRP & $>100 \mathrm{mg} / \mathrm{l}$ & $>10 \mathrm{mg} / \mathrm{l}$ \\
\hline Leukozyten & $>10000 / \mu \mathrm{l}$ & $>3000 / \mu \mathrm{l}$ \\
\hline PMN\% & $>90 \%$ & $>80 \%$ \\
\hline
\end{tabular}

Minor-Kriterien:

- erhöhtes Serum-CRP und erhöhte Blutsenkungsgeschwindigkeit (BSG)

- erhöhte Leukozytenzahl in der Synovialflüssigkeit ODER 2-fach ++ Veränderung auf dem Leukozytenesterasestreifen

- prozentual erhöhte polymorphkernige Neutrophile (PMN\%) in der Synovialflüssigkeit

- positive Histologie von periprothetischem Gewebe

- einzelne positive Kultur

Die entsprechenden Grenzen der Testresultate unterscheiden sich zwischen akuten und chronischen Infektionen ( $\triangleright$ Tab. 1).

\section{Periprothetische Infektdiagnostik}

Trotz des langjährigen Kampfes gegen die Infektion und der enormen Fortschritte existieren dennoch keine diagnostischen Verfahren, die mit einer 100-prozentigen Genauigkeit eine periprothetische Gelenkinfektion nachweisen oder ausschließen können. Dennoch gab es in den letzten 10 Jahren in der periprothetischen Infektdiagnostik wichtige Weiterentwicklungen. Die Punktion des betroffenen Gelenkes bleibt zwar weiterhin das wichtigste Verfahren zur Identifizierung des Keimes. Der Unterschied ist jedoch, dass man heutzutage die gewonnene Synovialflüssigkeit im Labor für die Bestimmung von Biomarkern nutzen kann. Die Punktion des betroffenen Gelenkes sollte idealerweise in einem Eingriffsraum oder ambulanten OP unter sterilen Kautelen und ohne die Nutzung eines Lokalanästhetikums erfolgen. Die bakterizide Wirkung des Lokalanästhetikums könnte zu falsch negativen Resultaten führen. Bei Vorhandensein eines Antibiotikums sollte dies entsprechend 14 Tage vor einer Gelenkpunktion abgesetzt werden.

\section{Biomarker in der periprothetischen Infektdiagnostik}

Deirmengian et al. haben im Rahmen einer Studie 43 Biomarker zur Diagnosestellung einer periprothetischen Gelenkinfektion untersucht [8]. Die Resultate dieser Studie zeigten, dass Alpha-Defensin eine 100-prozentige Sensi- 
tivität und Spezifität in der Diagnosestellung einer PPI aufwies. Alpha-Defensin, auch „human neutrophil peptide“ bezeichnet, wird von den neutrophilen Granulozyten sezerniert. Die Hauptfunktion von Alpha-Defensin ist die Zerstörung von Krankheitserregern durch Zerstörung ihrer Zellmembran. Das Alpha-Defensin kann entweder quantitativ oder qualitativ aus der gewonnenen Synovialflüssigkeit ermittelt werden. Für die Durchführung des quantitativen Alpha-Defensin-Tests ist ein Labor erforderlich, das eine ELISA-Immunassay-Untersuchung durchführen kann. Hierzu ist eine Probe von ca. 1 ml Gelenkflüssigkeit ausreichend. Aktuelle Studien weisen eine Sensitivität von 100\% und eine Spezifität von 96\% auf [28]. Der Alpha-Defensin-Test liefert konsistente Ergebnisse unabhängig vom Organismustyp oder der Virulenz des Organismus [9]. Ein Nachteil ist, dass die Resultate des quantitativen Tests frühestens nach 8 Stunden vorliegen. Daher wurde der qualitative Alpha-Defensin Lateral Flow Test (Synovasure, Zimmer, Warsaw, USA) auf den europäischen Markt gebracht. Dieses Testverfahren kann im OP oder entsprechend im Eingriffsraum unmittelbar nach erfolgreicher Punktion der Gelenkflüssigkeit erfolgen. Die Ergebnisse liegen nach ca. 10 Minuten vor. Dieser Alpha-Defensin-Schnelltest hat jedoch eine geringere Sensitivität und Spezifität als der quantitative Alpha-Defensin-Test [16,25]. Limitierende Faktoren beider Testverfahren sind die hohen Kosten und die Tatsache, dass keine Aussage über die Art des Keims und die Antibiotikaresistenz getroffen werden kann.

Der Leukozyten-Esterase-(LE-)Test ist hingegen ist ein kostengünstiges und einfach durchzuführendes Testverfahren. Die Testergebnisse liegen bereits nach 2 Minuten vor. Dieses Testverfahren wurde 2011 von Parvizi et al. für die Diagnosestellung einer periprothetischen Gelenkinfektion erstmalig demonstriert [22]. Aktuelle Studien weisen eine hohe Sensitivität und Spezifität auf [28]. Jedoch sind die Ergebnisse des LE-Testverfahrens dem quantitativen Alpha-Defensin-Test unterlegen [28]. Auch dieses Testverfahren gibt keine Aussage über die Art des Keims und die Antibiotikaresistenz.

\section{Therapieoptionen der periprothetischen Infektion}

Die Therapie zwischen einer akuten und chronischen periprothetischen Infektion unterscheidet sich enorm. Das Therapieziel bei einer Frühinfektion ist der Prothesenerhalt. Bei einer chronischen Infektion ist ein Prothesenerhalt jedoch nicht möglich und eine komplette Wechseloperation erforderlich. Kontraindikationen zum Prothesenerhalt bei Frühinfektionen sind ebenfalls zu berücksichtigen. Wenn die Möglichkeit für einen Wundverschluss aufgrund großer Weichteildefekte nicht besteht, ist ein Prothesenerhalt nicht möglich. Weitere Kontra- indikationen sind Fistelausbildungen und Prothesenlockerungen.

\section{Wundspülung und Débridement}

Die Wundspülung mit Débridement ist bei Patienten ohne allgemeine Sepsis kein Dringlichkeitsverfahren. Bei Patienten ohne eine begleitende Sepsis sollte daher der Zustand des Patienten vor dem chirurgischen Eingriff optimiert werden, um schwerwiegende intraoperative Komplikationen aufgrund bestehender Begleiterkrankungen zu vermeiden. Hier spielen Begleiterkrankungen wie eine unkontrollierte Hyperglykämie (>180 mg/dl), schwere Anämie ( $\mathrm{Hb}<10 \mathrm{mg} / \mathrm{dl})$ oder Gerinnungsstörung eine besondere Rolle.

\section{OP-Technik}

Der operative Zugang zum Gelenk sollte immer über die vorbestehende Wunde erfolgen. Wichtig für den Therapieerfolg ist, dass man ein aggressives Débridement durchführt, d. h. dass das komplette nichtvitale und infizierte periartikuläre Gewebe reseziert wird. Des Weiteren sollte eine komplette Synovialektomie erfolgen. Bei einer periprothetischen Infektion des Kniegelenkes ist vor allem die hintere Kapsel ausgiebig zu debridieren. Diverse Autoren empfehlen, im Rahmen einer Wundspülung und eines Débridements die modularen Teile der Prothese und das Polyethylen-Inlay auszutauschen [1-3, 26, 27]. Dies wurde auch im Rahmen des International Consensus Meetings für periprothetische Infektionen empfohlen $[12,13]$.

Ein kürzlich publizierte Multicenterstudie konnte zeigen, dass der Austausch der modularen Komponenten von Hüft- und oder Knieprothesen das Risiko für ein Versagen um 33\% reduziert [19]. Darüber hinaus ist die frühzeitige Entfernung aller modularen Komponenten wichtig, um die Bildung von resistenten Biofilmen und die Gesamtbakterienlast zu reduzieren. Im Rahmen des Débridements sollten 5-7 Proben aus allen relevanten Bereichen des Operationsgebietes für mikrobiologische und histologische Untersuchungen entnommen werden. Nach erfolgreichem aggressiven Débridement empfehlen wir eine entsprechende Wundspülung mit pulsatiler Spülung mit 0,02\% Polyhexanidlösung (Lavasept, B.Braun, Melsungen, Deutschland). Anschließend erfolgt ein schichtübergreifender Wundverschluss. Durchaus seltener heute genutzt, aber doch noch nicht ganz vergessen, ist die Anlage einer Saug-Spül-Drainage ( $\bullet \mathbf{A b b}$. 2). Eine SaugSpül-Drainage besteht i.d.R. aus 1-2 führenden Drainageschläuchen und 2 weiteren großlumigen abführenden Drainageschläuchen in Wund- oder Körperhöhlen, über die kontinuierlich eine Wundhöhle mit z.B. steriler Elektrolytlösung gespült wird, für maximal 48 Stunden. 


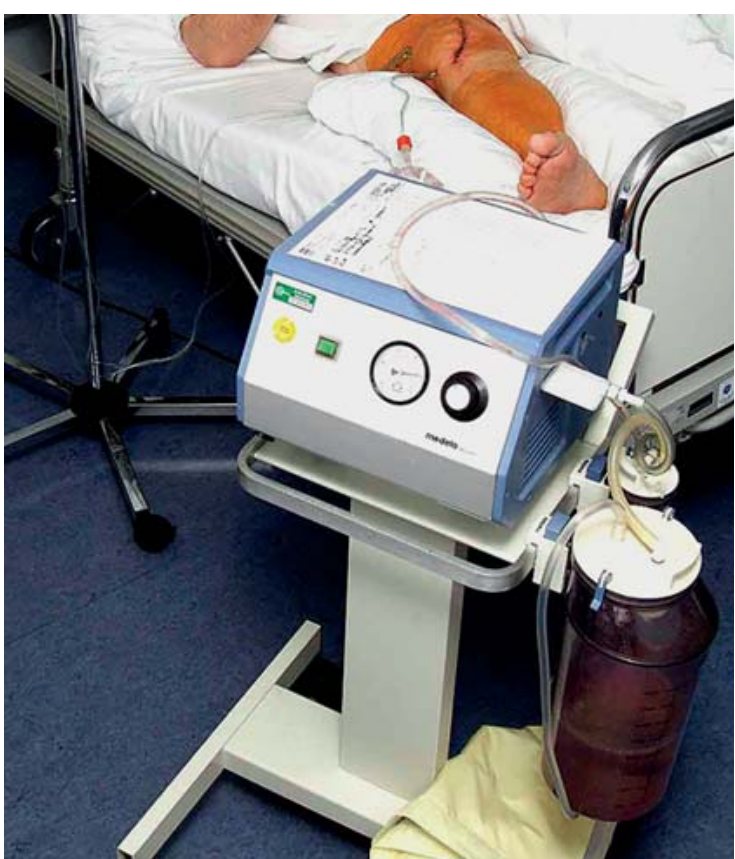

- Abb. 2 Anschluss einer Saug-Spül-Drainage an ein infiziertes Kniegelenk. Diese wird in der Regel für 48 Stunden belassen.

\section{Arthroskopische Wundspülung}

Die arthroskopische Behandlung ist aufgrund der Tatsache, dass sie weniger invasiv ist als die offene Wundspülung, ein attraktives Verfahren. Jedoch gibt es bisher nur wenige Studien, welche die Rolle der arthroskopischen Wundspülung und des Débridements in der Therapie einer periprothetischen Frühinfektion untersucht. 1998 berichteten Flood und Kolarik erstmals die erfolgreiche arthroskopische Wundspülung bei 2 akuten infizierten Knieprothesen [11]. Dixon et al. und Chung et al. berichteten, dass bei 9 von 15 Patienten (60\%) und 10 von 16 Patienten (62,5\%) eine Infektkontrolle erzielt werden konnte $[6,10]$. Während die Kniearthroskopie eine mögliche Rolle bei der Behandlung der periprothetischen Infektion gezeigt hat, ist die Arthroskopie des Hüftgelenkes für infizierte Hüftprothesen weniger gut beschrieben [15]. Dennoch ist zu erwähnen, dass die klinischen Resultate der arthroskopischen Wundspülung mit Débridement deutlich schlechtere Ergebnisse liefern. Der Chirurg kann mit einer Arthroskopie nicht auf alle Bereiche des Gelenkes zugreifen, um ein aggressives Débridement durchzuführen. Die arthroskopische Wundspülung mit Débridement spielt laut Konsensus-Empfehlungen der MSIS eines infizierten Prothesengelenkes keine Rolle [12, 13].

\section{Zusammenfassung}

Der Frühinfekt einer Endoprothese ist mittlerweile relativ klar definiert, wobei der Zeitraum nach Implantation oder dem Einsetzen erster akuter Symptome als wegweisend gilt. Infekte mit einem längeren Zeitraum als 4 Wochen sollten bereits als chronisch definiert werden. Konsequent sollte bei einem frühen Infekt ein Erhalt der Prothese angestrebt werden, bestehend aus einem aggressiven offenen Débridement, einer Spülung und dem Wechsel der mobilen Teile. Dies gilt für Hüft und Knieprothesen. Aufgrund der wesentlich schlechteren Ergebnisse spielt die rein arthroskopische Spülung heute keine Rolle mehr. Einer anschließenden zunächst i.v. Antibiose folgt in der Regel eine orale weiterführende Therapie für ca. 12 Wochen, je nach Erregerspektrum. Selbst bei diesem strukturierten Vorgehen liegt die Erfolgschance einer dauerhaften Eradikation des Infektes allerdings auch nur bei knapp 70\% laut aktueller Literatur. Bei chronischen Infekten sinkt bei diesem Vorgehen allerdings diese Erfolgsrate auf unter $20 \%$, sodass hier prinzipiell immer ein kompletter Prothesenwechsel erfolgen sollte.

\section{Interessenkonflikt}

Die Autoren erklären, dass kein Interessenkonflikt besteht.

Über die Autoren

Mustafa Citak

Priv.-Doz. Dr. med., Oberarzt und wissenschaftlicher Leiter, Orthopädische Chirurgie, Helios ENDO-Klinik Hamburg

\section{Akos Zahar}

Dr. med., Oberarzt, Orthopädische Chirurgie, Helios ENDOKlinik Hamburg

\section{Daniel Kendoff}

Prof. Dr. med., Chefarzt, Orthopädische Chirurgie, Helios Klinikum Berlin-Buch

\section{Korrespondenzadresse}

Priv.-Doz. Dr. med. Mustafa Citak

Oberarzt und wissenschaftlicher Leiter Helios ENDO-Klinik Hamburg

Holstenstraße 2

22767 Hamburg

Tel.: + 49403197 1663, Fax: + 494031971693

mustafa.citak@helios-kliniken.de 


\section{Literatur}

[1] Aboltins C, Dowsey MM, Peel T et al. Early prosthetic hip joint infection treated with debridement, prosthesis retention and biofilm-active antibiotics: functional outcomes, quality of life and complications. Intern Med J 2013; 43: 810-815

[2] Aboltins CA, Dowsey MM, Buising KL et al. Gram-negative prosthetic joint infection treated with debridement, prosthesis retention and antibiotic regimens including a fluoroquinolone. Clin Microbiol Infect 2011; 17: 862-867

[3] Aboltins CA, Page MA, Buising KL et al. Treatment of staphylococcal prosthetic joint infections with debridement, prosthesis retention and oral rifampicin and fusidic acid. Clin Microbiol Infect 2007; 13: 586-591

[4] Bauer TW, Parvizi J, Kobayashi $N$ et al. Diagnosis of periprosthetic infection. J Bone Joint Surg Am 2006; 88: 869-882

[5] Bozic KJ, Kurtz SM, Lau E et al. The epidemiology of revision total knee arthroplasty in the United States. Clin Orthop Relat Res 2010; 468: 45-51

[6] Chung JY, Ha CW, Park YB et al. Arthroscopic debridement for acutely infected prosthetic knee: any role for infection control and prosthesis salvage? Arthroscopy 2014; 30: 599-606

[7] Dale H, Hallan G, Hallan G et al. Increasing risk of revision due to deep infection after hip arthroplasty. Acta Orthop 2009; 80: 639-645

[8] Deirmengian C, Kardos K, Kilmartin P et al. Diagnosing periprosthetic joint infection: has the era of the biomarker arrived? Clin Orthop Relat Res 2014; 472: 3254-3262

[9] Deirmengian C, Kardos K, Kilmartin P et al. The Alpha-defensin Test for Periprosthetic Joint Infection Responds to a Wide Spectrum of Organisms. Clin Orthop Relat Res 2015; 473: 2229-2235

[10] Dixon P, Parish EN, Cross M]. Arthroscopic debridement in the treatment of the infected total knee replacement. J Bone Joint Surg $\mathrm{Br}$ 2004; 86: 39-42

[11] Flood JN, Kolarik DB. Arthroscopic irrigation and debridement of infected total knee arthroplasty: report of two cases. Arthroscopy 1988; 4: 182-186

[12] Haasper C, Buttaro M, Hozack W et al. Irrigation and debridement. J Arthroplasty 2014; 29 (Suppl. 2): 100-103

[13] Haasper C, Buttaro M, Hozack W et al. Irrigation and debridement. J Orthop Res 2014; 32 (Suppl. 1): S130-S135

[14] Hanssen AD, Osmon DR. Evaluation of a staging system for infected hip arthroplasty. Clin Orthop Relat Res 2002; 403: 1622

[15] Heaven $S$, de Sa D, Simunovic $N$ et al. Hip arthroscopy in the setting of hip arthroplasty. Knee Surg Sports Traumatol Arthrosc 2016; 24: 287-294

[16] Kasparek MF, Kasparek M, Boettner F et al. Intraoperative Diagnosis of Periprosthetic Joint Infection Using a Novel AlphaDefensin Lateral Flow Assay. J Arthroplasty 2016; 31: 2871 2874
[17] Kurtz S, Ong K, Lau E et al. Projections of primary and revision hip and knee arthroplasty in the United States from 2005 to 2030. J Bone Joint Surg Am 2007; 89: 780-785

[18] Kurtz SM, Ong KL, Lau E et al. Prosthetic joint infection risk after TKA in the Medicare population. Clin Orthop Relat Res 2010; 468: 52-56

[19] Lora-Tamayo J, Murillo O, Iribarren JA et al.; REIPI Group for the Study of Prosthetic Infection. A large multicenter study of methicillin-susceptible and methicillin-resistant Staphylococcus aureus prosthetic joint infections managed with implant retention. Clin Infect Dis 2013; 56: 182-194

[20] McPherson EJ, Woodson C, Holtom P et al. Periprosthetic total hip infection: outcomes using a staging system. Clin Orthop Relat Res 2002; 403: 8-15

[21] Oussedik SI, Haddad FS. The use of linezolid in the treatment of infected total joint arthroplasty. J Arthroplasty 2008; 23: 273-278

[22] Parvizi J, Jacovides C, Antoci V et al. Diagnosis of periprosthetic joint infection: the utility of a simple yet unappreciated enzyme. J Bone Joint Surg Am 2011; 93: 2242-2248

[23] Parvizi ], Pawasarat IM, Azzam KA et al. Periprosthetic joint infection: the economic impact of methicillin-resistant infections. J Arthroplasty 2010; 25 (Suppl. 6): 103-107

[24] Parvizi ], Zmistowski B, Berbari EF et al. New definition for periprosthetic joint infection: from the Workgroup of the Musculoskeletal Infection Society. Clin Orthop Relat Res 2011; 469: 2992-2994

[25] Sigmund IK, Holinka J, Gamper J et al. Qualitative $\alpha$-defensin test (Synovasure) for the diagnosis of periprosthetic infection in revision total joint arthroplasty. Bone Joint J 2017; 99: 6672

[26] Sukeik M, Patel S, Haddad FS. Aggressive early debridement for treatment of acutely infected cemented total hip arthroplasty. Clin Orthop Relat Res 2012; 470: 3164-3170

[27] Tsukayama DT, Estrada R, Gustilo RB. Infection after total hip arthroplasty. A study of the treatment of one hundred and six infections. J Bone Joint Surg Am 1996; 78: 512-523

[28] Wyatt MC, Beswick AD, Kunutsor SK et al. The Alpha-Defensin Immunoassay and Leukocyte Esterase Colorimetric Strip Test for the Diagnosis of Periprosthetic Infection: A Systematic Review and Meta-Analysis. J Bone Joint Surg Am 2016; 98: $992-$ 1000

[29] Zimmerli W, Trampuz A, Ochsner PE. Prosthetic-joint infections. New Engl J Med 2004; 351: 1645-1654

Bibliografie

DOI https://doi.org/10.1055/s-0043-102321

OP-JOURNAL 2017; 33: 136-140 @ Georg Thieme Verlag KC Stuttgart · New York ISSN 0178-1715 